\title{
El concepto de cultura en el derecho
}

\author{
The concept of culture in law studies
}

Manuel Alberto Jesús Moreira*

\begin{abstract}
Resumo: Neste trabalho apresenta-se a "cultura" como um conceito que permaneceu equivocado no campo do Direito, utilizado com significados confusos e subordinado a uma concepção evolucionista. O surgimento de novos direitos como o de "pluralidade cultural" e o de "plurietnicidade" estabeleceram um cenário litigioso sobre a operatividade jurídica do conceito. Desta maneira colocou-se em conflito a agência judicial com o processo reivindicativo que busca alcançar a igualdade mediante o tratamento desigual do diferente. Para entender este processo a partir do Direito deve-se definir o conceito de "cultura" despojando-o dos conteúdos evolucionistas e coloniais que se encontram ancorados na matriz disciplinar.
\end{abstract}

Palavras-chave: Direito; Cultura; Pluriculturalidade; Evolucionismo; Direitos individuais e coletivos

\begin{abstract}
The purpose of this report is to present the concept of culture which, because it has not received much attention recently in the field of Law Studies, it is used with confusing meanings and subordinated to an evolutionary conceptualization. The irruption of new rights through the concepts of 'cultural plurality' and 'pluriethnicity' has established a new belligerent scenario on this concept for juridical operativeness. In this way, the judicial agency has put itself in conflict within the vindictive processes that attempt to reach equality through an unequal treatment of difference. In order to understand this process from the field of Law, the concept of culture must be re-defined by freeing it from the evolutionary and colonial views still anchored in the disciplinary matrix.
\end{abstract}

Key words: Law; Culture; Pluriculturalism; Evolutionism; Individual and collective rights

\section{Sobre el concepto de cultura}

El concepto de "cultura" merece varias aclaraciones preliminares antes de pasar a explicar sus significados desde el Derecho. La voz "cultura" es unas de esas palabras que como una escalera larga y sinuosa nos conduce a una altura babélica que no termina en ninguna parte, pero que sin

* Doctor en Ciencias Jurídicas y Sociales (UNL), Magíster en Antropología Social (UNaM), Investigador Cedead, Posadas, Argentina.<moreira.arrechea@gmail.com>

\begin{tabular}{|c|c|c|c|c|c|}
\hline Civitas & Porto Alegre & v. 8 & n. 3 & p. $466-481$ & set.-dez. 2008 \\
\hline
\end{tabular}


embargo nos conecta en todas direcciones. Existen como niveles de usos y significados de la misma. En un primer nivel la vemos utilizada casi como una jerga, ya que se refiere a cualquier expresión, tanto de humor, de gusto musical, de vestimenta, de preferencia culinaria, de refinamiento intelectual o simplemente de estatus sociocultural y también de discriminación o exclusión. Desde esta perspectiva la cultura es como la máscara muy visible de un grupo humano, definida por los símbolos, descubierta por el uso o amplificada en el estereotipo. Es un comportamiento congelado.

En un segundo nivel la "cultura" se registra en el uso como una serie interdependiente de expresiones humanas. Entonces se combinan el idioma, las vestimentas, los símbolos, los rituales y la expresión artística como el soporte de los hábitos y comportamientos de una sociedad. La máscara se extiende y se conecta con otros fenómenos que asociados entre sí nos permiten descubrir un universo de identidades. Pero todavía flotamos sobre una superficie muy artificiosa. Solo vemos con nitidez la cultura material y la taxonomía material. Provisoriamente podemos establecer en esta perspectiva dos extremos: E. Tylor con su definición sostenida en un programa evolutivo y F. Boas con sus modelos culturales (patterns) sin evolución pero concentrada en un "particularismo histórico".

En el tercer nivel la idea de cultura abandona la materialidad y la esencialidad para internarse en una discusión que comprometió a varias disciplinas como la Lingüística, la Antropología y la propia Sociología. Desde la Antropología Social podemos observar de modo muy simplificado como C. Geertz (1999, p. 20-51) trató de explicarla desde una red de significados, o N. García Canclini (2006, p. 34) como el conjunto de los procesos sociales de significación, o para L. White Harris (1997, p. 550-563) una plataforma instrumental de adaptación. Esta somera enunciación no pretende más que revelar la contienda y preocupación teórica del debate que termina siendo una frontera metodológica. En realidad se trata de la última etapa de la discusión. Como si tropezamos con alienígenas y nos deja de preocupar de donde vienen para interesarnos exclusivamente en el contacto y si podemos comunicarnos amistosamente o verificar si sus intenciones son pacíficas.

La discusión sobre la cultura parece ensimismarse en la función y funcionamiento del "molde mental colectivo" en palabras de Kuper. Aunque por momentos también incluye los trastornos y el diagnóstico. El debate ha adoptado un rango metafísico y para ello fue abandonando todas las definiciones y acuerdos provisorios para sumergirse en la controversia sobre el proceso cultural. 
Aseguran algunos investigadores que resultaría muy difícil saber cuantas definiciones de cultura existen. En realidad el número no es tan importante, sino el tironeo epistemológico que fue contaminando ideológicamente la idea y luego apresada en fórmulas "esotéricas", lo que provocó mayores confusiones y desacuerdos. Con estos enredos la sensación de fracaso fue inevitable, porque ninguna definición quedó libre de alguna impugnación. Me pregunto antes de seguir: ¿Es definible la cultura o solamente podemos ejercitar una aproximación descriptiva? Quizá buscando un consenso provisorio Luciano Gallino recomienda (1995, p. 243): "será oportuno intentar trazar las coordenadas del amplísimo y polidimensional espacio conceptual, en cuyos diversos puntos se sitúan las principales definiciones de cultura". Es evidente que ese mapa no se puede confeccionar mediante coordenadas lógicas, porque la observación fue removida y desplazada por el interés en la función de la cultura. Parece más atractivo saber para qué existe la cultura y cómo funciona en los procesos de contacto que las ideas y valores. La labor descriptiva fue permutada por el cambio cultural, el proceso pluricultural y la función de los códigos simbólicos dentro de otros sistemas.

Ese estado beligerante de la discusión es un obstáculo difícil de remover a la hora de trasladar los significados de "cultura" al universo de los juristas que ven con aturdida perplejidad la invasión del derecho con la "diversidad cultural". Una tradición crítica interdisciplinaria que sin abandonar la ambigüedad conceptual se vuelca en el derecho con mayor incertidumbre.

El Derecho ha permanecido demasiado tiempo en un edén ilusorio, con paraíso, tierra prometida y expulsados. Dentro de un sistema de deberes, libertades y prohibiciones diseñado como un museo donde miramos las eras creciendo en el laberinto de la protohistoria hasta la humanización y la civilización. El "evolucionismo" no solo ha influido en el Derecho sino que se ha mezclado en su propia genética. Por este motivo la primera precaución es medir de qué manera vamos a trasladar los significados controvertidos, principalmente en la Antropología Social, para ser utilizados en la explicación de los nuevos derechos donde se incluye la idea de "cultura".

Y como segunda precaución es conveniente advertir si hablamos de "las culturas" o desde "la cultura". En el plural de la primera se encuentra contenido un problema que nos orienta hacia lo que fue denominado la multiculturalidad. En la segunda vemos en general nuestra cultura. De 
esa confrontación existen distinciones conceptuales como la de pluriculturalidad. ${ }^{1}$

Voy a desarrollar los principales aspectos de esta crisis epistemológica que suele conducirnos la investigación y el abordaje de cuestiones dentro de lo que se denomina interdisciplinariedad y el uso de conceptos prestados o malversados en el Derecho. En este caso el de "cultura".

\section{El uso de la cultura en el Derecho}

Los juristas utilizan el concepto de "cultura" en los dos primeros niveles anunciados, como un rasgo congelado o como un universo de expresiones suficientes para distinguir una identidad. En el peor de los casos la asimilan a la educación y sus distintos grados. También se asocia a una destreza, hábitos o costumbres.

La mayor de las veces el jurista propende a considerar a la cultura como el producto del progreso y en consecuencia a definir "la cultura" como un crecimiento histórico. Esa historicidad que el jurista asigna a la "cultura" se encuentra tabicada en etapas que determinan un estadio que va aumentando en complejidad y en mecanismos de control.

La idea que prevalece en el universo del Derecho es que la cultura es un producto de la evolución de la humanidad y se va perfeccionando como una tecnología. Los grupos étnicos y sociedades simples que no comparten este proceso quedan postergados en la historia, condenados al atraso primitivo. Para el Derecho presentan una incomodidad metodológica que solo puede remediarse imponiendo las reglas de la cultura dominante. Los principios y mecanismos del Derecho no resultan fungibles con otros principios que contradigan aquellos considerados como esenciales. Observemos la afirmación de Otfried Höffe (2000, p. 84) quien sostiene que la "presunción de inocencia" "que se expresa en el principio "in dubio pro reo' y que puede incluirse como parte del patrimonio de justicia de la humanidad. Podemos incluso sospechar que este principio esté reconocido en el enjuiciamiento criminal prácticamente desde que existe un derecho penal. Y solo en culturas muy arcaicas ... recae el peso de la prueba sobre el supuesto sacrílego ... ". En esta definición ya se advierte el etnocentrismo

\footnotetext{
R. E. Hamel (2001, p. 143-170) distingue entre "multiculturalismo" y "pluriculturalismo": En el primero la diversidad puede ser reconocida, inclusive como generadora de ciertos derechos, pero sigue siendo considerada como un problema, lastre o barrera para el desarrollo. En el segundo los segmentos más significativos de la sociedad no sólo reconocen la diferencia como derecho del grupo e individuo indígena, sino que la conciben, por el contrario, como un recurso sociocultural que enriquece el estado y el conjunto de la nación.
} 
legal que ni siquiera considera los estudios etnográficos que reportan formas judiciales donde el mismo principio puede modificarse por la presencias de lógicas diferentes. Así lo ha entendido Borja Jiménez (2001, p. 127-129) al comparar el derecho occidental y el de algunas comunidades indígenas señalando que: "Teniendo en cuenta estas realidades tan distintas, y la especial cosmovisión de determinadas etnias nativas, también la presunción de inocencia adquiere otra dimensión".

El evolucionismo jurídico nace con el evolucionismo antropológico, en alguna medida lo utiliza y aplica en los esbozos necesarios para explicar la mutación del Derecho. El principio fundamental era el progreso, que le hacía percibir que algunos pueblos habían avanzado más que otros. Lo hacían a través de modelos donde la sociedad Victoriana aparecía en la escala más alta. Asi H. Morgan (1980) clasificaba en salvajismo inferior (recolección de frutos silvestres), salvajismo medio (captura del pescado, uso del fuego y lenguaje), salvajismo superior (utilización del arco y la flecha), barbarie inferior (invención de la cerámica), barbarie media (domesticación de las plantas y animales) barbarie superior (utilización de armas y el metal) y civilización (invención de la escritura). Lo mismo reproducía H. Maine (1977, p. 13-15) cuando hablaba de estadios que iban desde el contrato al código escrito. Tampoco Marx y Engels pudieron escapar a esa fascinación antropológica y utilizaron el programa evolutivo de Morgan para probar el origen del comunismo. Y en la Ideología alemana asimilan ideología con cultura con el mismo soporte material y determinista donde incluyen creencias, derecho y religión (Marx, Engels, p. 29-32).

La historia del Derecho categoriza a pueblos "salvajes" y "primitivos", para describir un remoto antepasado que en una accidentada metamorfosis forense culmina en los derechos humanos. La idea de progreso y evolución se conforma con patrones ideales que petrifican la justicia a la vez que facilitan la desigualdad. En ese trayecto evolutivo la oralidad se transforma en códigos, los derechos colectivos en criptas individuales, el juzgamiento popular en jueces unipersonales y las sanciones difusas en un catálogo de mortificantes formas punitivas. Esta parece ser una buena síntesis del tránsito de lo "primitivo" a la "civilización". La noción evolutiva en el Derecho se fortaleció con la consulta a dudosas etnografías, de las que los juristas extrajeron modelos allí descriptos para compararlos con los propios sistemas jurídicos, en una operación deficiente científicamente.

Un ejemplo de las prácticas etnográficas de gabinete lo constituye la obra La rama dorada de J. G. Frazer, quien sin abandonar su escritorio fue describiendo culturas que iba conociendo con documentos y crónicas 
de viaje. Allí se exponían costumbres y rituales que obviamente en una confrontación elemental y no científica podían producir la sensación de niveles de humanidad, como fósiles culturales divididos en ritmos evolutivos. Ese paradigma de la evolución es rescatada por numerosos juristas que usaron esas descripciones etnográficas para comparar y establecer una historia del derecho lineal, que arranca en formas defectuosas y bárbaras, dentro de prácticas orales para culminar en el código escrito. A. Kuper (The invention of primitive society) mencionado por Fitzpatrick (1998, p. 107) decía que "el estudio de la sociedad primitiva no se consideraba, por lo que general, como una rama de la historia natural. Fue tratado inicialmente como una rama de los estudios jurídicos".

El Derecho occidental en sus orígenes se presenta como una disciplina dominante donde se establecen los patrones, las reglas, los cánones e inclusive se ordenan los significados en códigos que orientan ideológicamente. No es casual que los primeros antropólogos hayan sido abogados (Bachofen, Morgan, McLennan y Maine) lo que de alguna forma explica esta convergencia disciplinar transitoria.

Pero la Antropología Social se emancipa y decide cambiar sus paradigmas y rompe con el evolucionismo a partir de Franz Boas y su mirada "particularista histórica", cosa que no ocurre con el Derecho que extiende sus programas evolucionistas a todas las nuevas ramas jurídicas. Si observamos rápidamente textos de derecho penal, los juristas, al menos su gran mayoría describen los institutos legales como resultado de una evolución. En general todos los tratados de Derecho Penal incorporan un capítulo para contar la historia de un derecho bárbaro, mágico y cruel. El paradigma de la racionalidad usa el contraste siempre eficaz de "salvaje" y "civilizado". M. Foucault (2006) y luego L. Wacquant (2000) se han encargado de desmitificar prolijamente esa idea de evolución y progreso del Derecho Penal cuyo "apogeo" comienza con la tecnología carcelaria y termina en el "social-panoptismo".

La crisis del paradigma ocurre cuando irrumpen en el escenario nuevos derechos e ideas que deben explicarse a luz de renovados esquemas conceptuales. Y que solo pueden aceptarse mediante la intervención multidisciplinaria y construirse a partir de categorías cuyos significados se oponen belicosamente a los aceptados tradicionalmente. Por ejemplo "pluralidad cultural", "derechos indígenas", pluralidad jurídica" y "derechos colectivos".

La idea de "cultura" fue considerada durante mucho tiempo sin valor operativo dentro del derecho. Utilizada como equivalente a costumbre, medios de vida, hábitos o creencias como elementos sociales fosilizados. 
Ese significado superficial asignado a un ritual social, o al conocimiento de una clase social no se modifico y permaneció congelado en la conciencia legal. Para el Derecho la "cultura" era una cualidad de un sujeto social por el que se le asignaba un mayor o menor estatus. No producía efectos jurídicos determinantes. Era un registro lateral que permitía mensurar la pena, deportar, internar o designar tutores.

Su irrupción en la dimensión jurídica vino disfrazada en el interior del cambio de paradigmas constitucionales. Primero desapercibida detrás de todo lo "plural" que se agitaba como una retórica de libertad. El tono belicoso posterior reveló que las "culturas" no eran solamente la conexión con los nuevos paradigmas, sino que se trataba de un nuevo paradigma.

Es importante advertir que el concepto de "Cultura" es novedoso para nuestro derecho positivo y reciente en los ordenamientos legales comparados. No es mencionado en el Código Penal Argentino, no obstante la importancia que tiene en la mensuración de la pena. No es mencionado en el Código Civil aún cuando la reforma constitucional y el Convenio 169 modifican la idea tradicional de propiedad. Sin embargo la Reforma de la Constitución de 1994 la incluye repetidamente. En el art. 75 inc. 17 "se reconoce la identidad étnica y cultural de los pueblos indígenas argentinos... se garantiza la educación bilingüe e intercultural..." En el inc. 19 in fine dice "Dictar leyes que protejan la identidad y pluralidad cultural..." y en el art. 22 en el reconocimiento jerárquico de los tratados internacionales sobre derechos humanos.

Durante la Convención Constituyente de 1994 al tocar el tema de los derechos culturales comenta Alberto Castells (1995, p. 108) que hubo posicionamientos crípticos y difusos. El resultado de la votación de la sesión ordinaria del 10 de agosto fue de 184 votos por la declaración de la nación "plurietnica" y "pluricultural" y 84 votos por la negativa, quedando sin registrar 6 votos. Esto nos indica que un tercio de los representantes votó por la hegemonía cultural, manteniendo la visión colonial. Tal resultado nos permite presumir que a gran parte de la sociedad argentina le despierta rechazo la idea de aceptar la diversidad cultural como una amenaza a los valores tradicionales.

También fue incluido el concepto "cultura" en numerosos documentos internacionales, elaborados, sin duda por equipos multidisciplinarios existentes en los organismos internacionales, como lo fueron el Convenio 169 de la OIT y otros relacionados con los derechos de las minorías y grupos étnicos. 
Max Ernest Maier (2000, p. 45-47) jurista alemán de principios del siglo pasado postulaba la distinción entre "normas jurídicas" y "normas de cultura", llegando a la ingeniosa conclusión de que la primera estaba dirigida a los jueces y la segunda al pueblo. Para sostener su hipótesis construye un silogismo muy elástico donde "norma" es tanto la ley escrita (jurídica) como las prohibiciones religiosas y morales (cultural). De esta manera creaba una artificiosa clasificación. Los significados y la descripción son deficientes pero los datos son verdaderos. Es evidente que quienes aplican la ley deben conocerla mejor, como que también el pueblo ignora o conoce defectuosamente la ley. Si utilizamos estas evidencias como universos independientes resulta obvia la conclusión. Pero también es obvio el orden falso de la conclusión. En realidad la distinción solo puede ser discutida en los sistemas con derecho escrito, porque en los sistemas orales como el derecho indígena carece prácticamente de sentido. De todos modos la distinción presenta al derecho como un fenómeno artificialmente instalado, copia incompleta del derecho cultural ya existente. Y esa apertura metodológica justifica la discusión teórica y convierte a Maier en precursor del debate sobre la "cultura" como fundamento del derecho.

$\mathrm{Y}$ es un antecedente que vale la pena tener en cuenta porque en muchos documentos elaborados por expertos se recomienda observar el impacto cultural. Así en los casos de los indígenas que pretenden reclamar sus derechos ante la agencia oficial los expertos Yrigoyen F. y Ferrigno F. (2003, p. 6) concluyen que el acceso a la justicia debe ser "del modo cultural y lingüísticamente adecuado". Condición que adelanta un presupuesto tendiente a garantizar la igualdad ante la ley y la eficacia del servicio de justicia.

La agencia judicial disponible no debería favorecer exclusiva y selectivamente a quienes conozcan sus significados y funcionamiento; dificultando el acceso a quienes provengan de culturas diferentes o hablen idiomas distintos al oficial. Ya que de ser así el acceso se malograría por afectar un principio superior que es el de la igualdad.

La interacción judicial puede verse comprometida por diferencias de "culturas" entre quien reclama y el agente judicial. La cultura es un vehículo que facilita o dificulta la interacción judicial, generando desacuerdo, error cognitivo, impermeabilidad del sistema ante la rigidez cultural y prejuicio de sus operadores. También inadecuación de los métodos conocidos dentro de un sistema cultural desconocido, donde la producción de signos y valores puede resultar adversamente interpretada, ante la ausencia de una 
preparación idónea del operador para tratar situaciones que se apartan de los estándares conocidos.

El propio estado nacional to ha reconocido en un documento denominado "Plan Nacional contra la discriminación" (2005, p. 33-34) al detectar fallas en el sistema que produce diferentes modos de negar la alteridad. Para evitar la desigualdad en el acceso a la justicia recomienda entre sus propuestas ( $\mathrm{n}^{\circ}$ 60): “Arbitrar los medios para capacitar y actualizar los contenidos de la formación de jueces, operadores de justicia y funcionarios públicos sobre el desarrollo de la normativa y compromisos internacionales de nuestro país en materia de derechos humanos y comprensión de los mecanismos de la discriminación en todas las esferas" (2005, p. 215).

En un trabajo reciente (Moreira, 2008, p. 206) comentaba que en realidad el detonador de la discriminación se halla en un inventario cognitivo, especie de mapa mental construido con anterioridad al acto que declara la desigualdad. Se encuentra pautado en el sistema cultural colonial, que se compone de las creencias y representaciones más refractarias de los operadores estatales. No aparece como un acto discrecional o arbitrario del agente discriminador. Tampoco es posible comprobar que ese agente sabe que está cometiendo un acto prohibido, sino que obra con la convicción de estar trabajando en defensa de un catálogo de estereotipos donde figuran seres abstractamente considerados enemigos del sistema.

\section{Cambio de paradigmas e impacto legal del concepto "cultura"}

La adopción de conceptos y nomenclaturas de la Antropología Social dentro de las reformas constitucionales americanas, en los códigos de procedimientos penales y en los propios documentos internacionales adoptados por los estados nos obligan a explorar sus significados. Sobre todo es necesario comprender que estas nuevas herramientas arrastran una fuerte connotación ajena al derecho por lo que exige una mirada multidisciplinaria.

También en la labor de Naciones Unidas, en los documentos sobre derechos de las minorías, no discriminación, contra el racismo, derechos lingüísticos, diversidad cultural, pluralidad jurídica y pueblos indígenas, los cambios de paradigmas son muy notorios, y son admitidos a partir de categorías que provienen de otras disciplinas.

La Constitución de México de 2001 en el art. 4 dice: "la nación mexicana tiene una composición pluricultural sustentada originalmente en sus pueblos indígenas que son aquellos que descienden de poblaciones 
que habitaban en el territorio actual del país al iniciarse la colonización y que conserva sus propias instituciones sociales, económicas, culturales y políticas o parte de ellas...El derecho de los pueblos indígenas a la libre determinación se ejercerá en un marco constitucional de autonomía que asegure la unidad nacional...". La de Bolivia de 2004 indica: art. 1 Bolivia, libre, independiente, soberana, multiétnica y pluricultural...". La de Brasil 1988-2002 le dedica un capítulo a la cultura donde señala: El estado protegerá las manifestaciones de las culturas populares, indígenas y afrobrasileras, y de otros grupos participantes del proceso civilizatorio nacional". La de Canadá de 1982 consagra lo siguiente en la sección 27: "Esta carta será interpretada de una manera consistente con la idea de preservación de la herencia multicultural de los canadienses..." La de Colombia de 1991-2001 indica en su art. 7 "El estado reconoce y protege la diversidad étnica y cultural de la nación colombiana". Y luego en su art. 68 "Los integrantes de los grupos étnicos tendrán derecho a una formación que respete y desarrolle su identidad cultural". La de Ecuador de 1998 en su art. 1 declara: "El Ecuador es un estado social de derecho, soberano, unitario, independiente, democrático, pluricultural y multiétnico". Luego en sección VI dedicada a la cultura completa en el art. 62 "La cultura es patrimonio del pueblo y constituye elemento esencial de su identidad. El estado promoverá y estimulará la cultura... así como del conjunto de valores y manifestaciones diversas que configuran la identidad nacional, pluricultural y multiétnica". Guatemala 1985/1993 en la sección II dedicada a la cultura proclama en el art. 58: "Se reconoce el derecho de las personas y de las comunidades a su identidad cultural de acuerdo a sus valores, su lengua y sus costumbres". Honduras de 1982/1999, ordena en su art. 173 "El estado preservará y estimulará las culturas nativas, así como las genuinas expresiones del folclore nacional, el arte popular y las artesanías". Nicaragua en la reforma de 1995, en su art. 91 declara: "El estado tiene la obligación de dictar leyes destinadas a promover acciones que aseguren que ningún nicaragüense sea objeto de discriminación por razón de su lengua, cultura y origen". La de Panamá de 1994 en el capítulo IV, en el art. 83: "El estado reconoce que las tradiciones folclóricas constituyen parte medular de la cultura nacional y por tanto promoverá su estudio, conservación y divulgación, estableciendo su primacía sobre manifestaciones o tendencias que la adulteren". La de Paraguay de 1992 establece en el art. 62: "Esta Constitución reconoce la existencia de los pueblos indígenas, definidos como grupos de cultura anteriores a la formación y organización del estado paraguayo..." En el art. 140 señala: "El Paraguay es un país pluricultural y bilingüe". La Constitución 
de Perú de 1993 consagra en su art. 2 que "Toda persona tiene derecho a su identidad étnica y cultural. El estado reconoce y protege la pluralidad étnica y cultural de la nación...”. En la de Venezuela de 1999 en el capítulo VI que titula De los derechos culturales y educativos enuncia en su art. 100 "Las culturas populares constitutivas de la venezonalidad gozan de atención especial, reconociéndose y respetándose la interculturalidad bajo el principio de igualdad de las culturas".

Como se puede advertir en una década se desata en América una avalancha de nuevos derechos anunciando un cambio de paradigmas: la pluriculturalidad, la diversidad, y los derechos lingüísticos. Bajo el paraguas de la igualdad son derechos que modifican el imaginario legal, rompen el molde colonial y sacuden definitivamente la idea rígida del evolucionismo enquistado en el Derecho.

En este punto resulta importante detenernos brevemente en el pensamiento de Giovanni Sartori, quien distingue entre "diversidad" y "multiculturalidad" habilitando con su interpretación una de las polémicas más ásperas. Según Sartori (2001, p. 61-62) el problema consiste en considerar al multiculturalismo como un valor. Según este autor si una sociedad no es heterogénea no hay necesidad de multiculturizarla. El mejor de los mundos no será aquel en una diversificación eternamente creciente. Con la pregunta “'hasta que punto la tolerancia debe ser tolerante?” se juridiza el concepto, porque Sartori comienza a ubicar a la sociedad hegemónica dentro una dogmática evolucionista. Entonces casi resulta inevitable sospechar detrás de sus palabras las ideas de asimilación y de integración. Estamos de nuevo frente al "derecho civilizador". Sobre esta posición ideológica comenta H. Vázquez (2004, p. 131) que Sartori "confunde, desdibuja y banaliza la lucha por el reconocimiento, la identidad y los derechos diferenciados en las que se han comprometido los movimientos étnicos, cuyos miembros conviven cotidianamente en el seno de sociedades que los discriminan".

Con la ficción doctrinaria de que el estado es el único productor del derecho, como que también la ley se presume conocida por todos o que no existen formas jurídicas fuera de la legalidad estatal, se encuentra concebida una evidente artificialidad, empobrecida por los dogmas y amordazada por la intransigencia ideológica.

El trabajo multidisciplinar solo resulta verdaderamente útil cuando se ve a la disciplina convergente o las categorías que se toman prestadas con sus significados originales y sin perder de vista las matrices disciplinares desde donde fueron construidas. Caso contrario solo aparecerían dudosos préstamos, mecanismos extravagantes para presentar el análisis como 
novedoso o aún para legitimar métodos particularmente diferentes a los contenidos conceptuales.

En el ámbito del derecho, durante mucho tiempo se consideró una especie de profanación introducir métodos, categorías o resultados alcanzados en otras disciplinas. Cuando se mencionaban estas cuestiones que rozaban o mezclaban otras tradiciones académicas se las reinterpretaba desde el derecho, como una expropiación legitima, porque el marco jurídico codificado clausuraba otra posibilidad. Esta creencia de un monismo reduccionista y monopólico en la asignación de significados fue la ficción colonial y hegemónica necesaria para la construcción de mentalidades monoculturales.

La necesidad de ver la realidad y abandonar el molde dogmático para internarse dentro de las sinuosidades sociales implica un desafío que conduce al jurista a ver muchas cosas diferentes, la primera es la de poner en duda los tópicos fundacionales del derecho conectando lo individual a lo colectivo. Detrás de los "derechos colectivos" se oculta la idea de "cultura" y esa herramienta conceptual facilita la descripción del objeto controversial pero al mismo tiempo dificulta la ubicación del adjetivo "colectivo". Porque no permite divisar con familiaridad al "titular del derecho" o al "derecho" propiamente dicho. Estas confusiones suceden porque el impacto legal alcanzó al propio lenguaje jurídico y fue afectando de distintas maneras las herramientas analíticas del modelo legal.

Para evitar esta ambigüedad semántica es necesario expandir el campo disciplinar y exportar los significados con una cautelosa reserva científica de modo de evitar en el nuevo programa cognitivo discusiones que debiliten la adaptación de estos nuevos recursos jurídicos. La mutación conceptual del derecho no ha sido solo una retórica, sino que se destrabaron las fronteras dogmáticas cómodamente alambradas por los códigos.

Las categorías prestadas, deberían ser utilizadas no en función de las categorías conocidas, sino desde las pautas científicas desde donde fueron construidas. Porque si hablo de "pueblos indígenas", "derechos indígenas", "cultura indígena" y luego mezclo con grupos evolucionados y no evolucionados, estoy modificando las herramientas científicas utilizadas. También lo haré si hablo de "derechos colectivos" sin desprenderme del credo de "derechos individuales".

El nuevo horizonte de derechos, reivindicaciones y reconocimientos va asociado a la adopción de categorías escasamente exploradas en el derecho por lo que resulta necesaria mucha precaución científica a la hora de tratar temas que exceden el marco del derecho y que antes de este cambio de 
paradigmas eran considerados muy superficialmente. No se puede simplificar en fórmulas normativas la densidad epistemológica del objeto principal de estudio de la Antropología Social.

Muchos fenómenos actuales, procesos sociales, anomia, escenarios de conflictos que deben ser resueltos judicialmente encuentran herramientas analíticas dentro de la Antropología Social. La nueva terminología que aparece dentro del campo jurídico, como "derechos étnicos", "cultura", "pluralidad cultural", "interculturalidad" entre otros, merecen una atención especial y un tratamiento multidisciplinario.

Y este desafío implica otras razones y preguntas apremiantes sobre el Derecho en su condición de saber científico. Porque en la medida en que diseñe sus programas como una Ciencia debe prestar más atención para definir con exactitud las categorías y aplicar los marcos teóricos que provienen de otras disciplinas.

No existen dudas que las creencias dominantes en el ámbito legal compartido por juristas, abogados y operadores judiciales se encuentran contaminadas por la idea evolucionista de la cultura. Pero paradójicamente los documentos, declaraciones de derechos y jurisprudencia de los tribunales internacionales apuntan en dirección contraria. La contradicción permite suponer una anomia estructural porque se sigue pensando en el modelo anterior, pero dentro de un modelo pluricultural. Esta evidencia hace necesario revisar los programas de la cultura hegemónica que vacila y demora en aplicar la nueva legalidad.

\section{Conclusiones}

Los desafíos más grandes para el Derecho actual comienzan por aceptar y entender la existencia de una nueva axiología jurídica, la sustitución de los paradigmas del sistema liberal y el redescubrimiento de las clases y pueblos oprimidos por ese mismo derecho. Se trata de una cuestión de interpretación, de apertura axiológica y renovación paradigmática.

Si seguimos mirando el Derecho desde una concepción evolucionista va a resultar muy difícil desarticular la ideología liberal que considera la individualidad como el único sujeto de derechos. La tendencia de suponer a lo individual como la garantía suprema es tan negativa como colectivizar todos los derechos.

En el caso de los "derechos colectivos" se han creado barreras disciplinares, que Cruz Parcero (2007, p. 101-126) con simplificada economía se ha encargado de explicar señalando los errores de continuar 
obstinadamente dentro de los significados disciplinares propios. Citando a Hartney dice el autor que el adjetivo "colectivo" "sirve para identificar una clase de derechos sobre la base de otras características en vez de la naturaleza del titular del derecho" (Hartney, 1991, p. 114).

Con la misma evidencia lógica que se nos aparece en el horizonte social los "derechos colectivos" en el campo jurídico se advierte su incomodidad legal. No solamente es producto de la intransigencia dogmática sino además existe una cuestión técnica. Es necesario desarmar todo el sistema legal de occidente para hallar una explicación a su funcionamiento. Y la vía más acertada indica una urgencia preliminar: acordar los significados del concepto "cultura". Porque justamente en la "cultura" se encuentra la bisagra que conecta los dos modelos, el individualista al colectivista. No como una contienda sino como un complemento.

Con los "derechos colectivos" son básicamente tres dimensiones las que ingresan al derecho con estridencia retórica pero con torpeza operativa. Porque todo el sistema normativo de occidente se preparó para reconocer al individuo como único sujeto o principal portador de derechos. Y esas tres dimensiones son la idea de propiedad (territorio colectivo y hábitat), las libertades políticas (autodeterminación y métodos jurídicos) y el idioma (bilingüismo). Definirlas y aplicarlas exigirá una cuota muy grande de sistematización legal y una revisión completa del modelo. Aún de los "derechos humanos".

Explica Sousa Santos (2001, p. 296-297) que la vida socio-jurídica de fin de siglo se encuentra constituida por la intersección de diferentes fronteras jurídicas. Estas fronteras porosas constituyen la interlegalidad. La cuestión principal es saber quien es el sujeto de esa interlegalidad. Para identificarlo rechaza la idea del individuo abstracto de la teoría jurídico-política liberal como sujeto universal de derechos. El sujeto de la interlegalidad - concluye Sousa Santos - es una configuración de subjetividades. Con esa premisa sugiere: "Entre el individualismo y el colectivismo propongo el colectivismo de la subjetividad como una de las vías posibles de construcción de una nueva teoría de la subjetividad jurídica". Esta nueva configuración es la tarea encomendada a los juristas. Como un programa que agotó su rendimiento y es necesario reemplazarlo.

La conquista de los "derechos individuales" no debería confrontarse con los "derechos colectivos" que se encuentran activados en los documentos internacionales porque estos no suprimen lo individual sino que lo enriquecen. Se socializa el derecho en un contexto de alteridad. El propio credo de los derechos humanos contiene una saturación de individualismo 
y luego entra en crisis con el diseño de los "derechos colectivos". Supiot (2005, p. 266-267) ha considerado que es necesario explorar y superar el "fundamentalismo" de los derechos humanos que funciona como dogma y se reproduce como un credo. Para desactivar el fundamentalismo individualista es necesario interpretar adecuadamente la "cultura" desde el derecho.

La "cultura" como categoría operativa dentro del derecho deberá restablecer el marco que defina la fluidez legal necesaria para garantizar los derechos de los grupos minoritarios, los pueblos originarios y toda forma de alteridad rescatada a un plano de igualdad. Definir la "cultura" desde el derecho, sin el etnocentrismo legal es una tarea ineludible para aceptar la diferencia y articular adecuadamente la nueva legalidad a una sociedad plural, definitivamente igualitaria. El Derecho requiere de un profundo cambio estructural que unifique todos sus campos con los nuevos principios, rescatando la "pluralidad cultural" como el colectivismo de la subjetividad.

\section{Referencias}

BOAS, Franz. Cuestiones fundamentales de Antropología cultural. Buenos Aires: Ediciones Solar-Librería Hachette, 1964.

BORJA JIMÉNEZ, Emiliano. Introducción a los fundamentos del Derecho Penal Indígena. Tirant lo Blanch, Valência, 2001.

CANCLINI, Nestor Garcia. Diferentes, desiguales y desconectados: mapas de la interculturalidad. Barcelona: Gedisa, 2006.

CASTELLS, Alberto. El derecho de las culturas en la nueva Constitución Nacional. En: Estudios sobre la reforma constitucional de 1994. Instituto de Investigaciones Jurídicas y Sociales “Ambrosio L. Rioja”, y Ediciones Depalma, 1995.

CRUZ PARCERO, Juan Antonio. El lenguaje de los derechos: ensayo para una teoría estructural de los derechos. Madrid: Editorial Trotta, 2007.

FITZPATRICK, Peter. La mitología del derecho moderno. México: Siglo XXI Editores, 1998.

FOUCAULT, Michel. Vigilar y castigar: nacimiento de la prisión. Buenos Aires: Siglo XXI Editores, 2006.

FRAZER, James George. La rama dorada. México: Fondo de Cultura Económico, 1998.

GALLINO, Luciano. Diccionario de Sociología. México: Siglo XXI Editores, 1995.

GEERTZ Clifford. La interpretación de las culturas. Barcelona: Gedisa, 1999.

HAMEL, Rainer Enrique. Politicas del lenguaje y educación indígena en México. Orientación cultural y estrategias pedagógicas en una época de globalización. In: BEIN, Roberto; BORN, Joachim, Politicas lingüisticas: norma e identidad. Buenos Aires: UBA, 2001. 
HARRIS, Marvin. El desarrollo de la teoría antropológica: historia de las teorías de la cultura. México: Siglo XXI Editores, 1997.

HÖFFE, Otfried. Derecho intercultural. Barcelona: Gedisa Editorial, 2000.

JAKOBS, Günther. Derecho penal del enemigo: concepto jurídico-penal de acción en la dogmática contemporánea. Córdoba: Editorial Mediterránea, 2007.

MALINOWSKI, Bronislaw. Una teoría científica de la cultura y otros ensayos. Buenos Aires: Editorial Sudamericana, 1978.

MAINE, Henry. Ancient law. Dent: London, Melbourne and Toronto, Everyman's Library, Dutton; New York, 1977.

MAIER, Max Ernest. Normas jurídicas y normas de cultura. Buenos Aires: Hammurabi - Jose Luis Depalma Editor, 2000.

MARX, Karl; ENGELS, F. Ideología alemana. v. 1. Feuerbach: contraste entre el materialismo y el idealismo. Buenos Aires: Need, 1999.

MOREIRA, Manuel. Normalidad burocrática y discriminación. In: Investigaciones por la diversidad. Buenos Aires: Ministerio de Justicia, Seguridad y Derechos Humanos, Inadi, 2008.

MORGAN, L. La sociedad primitiva. Bogotá: Editorial Ayuso-Pluma, 1980.

PLAN NACIONAL CONTRA LA DISCRIMINACIÓN. Presidencia de la Nación. Boletín Oficial de la República Argentina, 27 de setiembre de 2005, año CXIII, $\mathrm{n}^{\mathrm{o}}$ 30.747, Buenos Aires.

SARTORI,Giovanni.Lasociedadmultiétnica:pluralismo,multiculturalismoyextranjeros. Madrid: Editorial Taurus, 2001.

SOUSA SANTOS, Boaventura de. El estado y el derecho en la transición posmoderna: por un nuevo sentido común sobre el poder y el derecho. In: COURTIS, Christian. Desde otra mirada: textos de teoría crítica del Derecho. Buenos Aires: Eudeba, 2001.

SUPIOT, Alain. Homo juridicus: ensayo sobre la función antropológica del Derecho. México: Siglo XXI Editores, 2007.

VAZQUEZ, Héctor. Antropología emancipadora, derechos humanos y pluriculturalidad. Rosario: Homo Sapiens Ediciones, 2004.

YRIGOYEN FAJARDO Raquel; FERRIGNO FIGUEROA, Víctor. Acceso a la justicia en Guatemala: situación y propuestas. Guatemala, mayo de 2003.

WACQUANT, Loïc. Las cárceles de la miseria. Buenos Aires: Manantial, 2000.

Recebido em: 26 jun. 2008 Aprovado em: 11 set. 2008 